\title{
Performance Evolution of Ad-hoc Routing Protocol with Shadow Propagation Using NS2
}

\author{
Er. Ajay Sharma \\ Student (M.Tech), CSE, India
}

\begin{abstract}
This Paper explained the performance evolution of ad-hoc routing protocols with shadow propagation i.e. AODV, AOMDV and DSDV in specific simulation situations and observing their conduct in phrases of 3 enormous parameters i.e. packet shipping fraction, and gen throughput in order to discover which one need to be preferred whilst the mobile ad hoc network needs to be set up for the precise duration underneath special situations. After implementing the three routing protocols under different propagation, the different conclusions have been drawn and results are optimized.
\end{abstract}

Keywords: AODV, AOMDV, NS2, DSDV, Routing

\section{INTRODUCTION}

In view that their emergence inside the Seventies, wireless networks have come to be more and more popular in the computing enterprise. That is mainly proper within the past decade, which has visible wireless networks being adapted to enable mobility. A mobile unit within these networks connects to, and communicates with, the closest base station this is within its conversation radius. As the mobile travels out of variety of one base station and into the variety of some other, a "handoff" happens from the vintage base station to the brand new, and the mobile is capable of keep verbal exchange seamlessly all through the network. Common applications of this type of network include workplace Wi-Fi nearby location networks (WLAns).
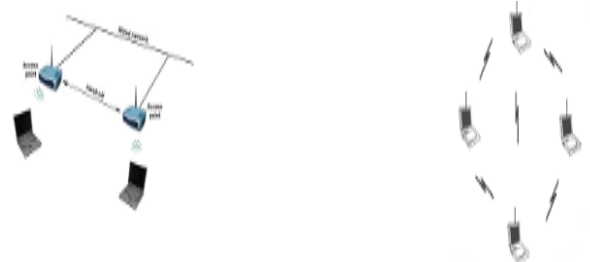

Fig 1 Infrastructure Based Wireless Network

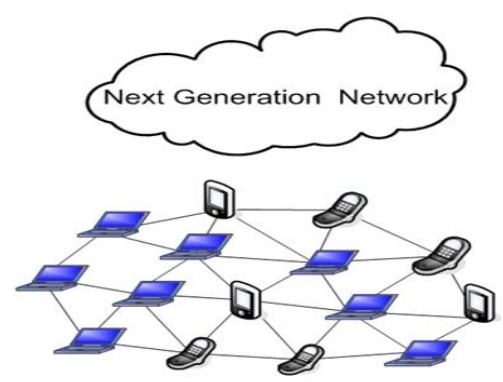

Fig 2 MANET Infrastructure (Karthik et al, 2010)

The fundamental difference between fixed networks and MANET is that the computers in a MANET are mobile. Fast set up of a conversation infrastructure at some point of a natural/environmental disaster that demolished the previous conversation infrastructure.

\section{Routing protocols in MANET:}

Routing is an act of moving information across an internetwork from a source node to the destination node. Routing is responsible for producing routes that meet the service requirements of the traffic session with in the service constraints imposed by the network. Routing protocols are wished whenever added statistics packets want to be surpassed over several nodes to reach at their locations. Routing protocols should find routes for packet transport and make certain the packets are added to appropriate destinations. 
Vol. 8, Issue 1, January 2019

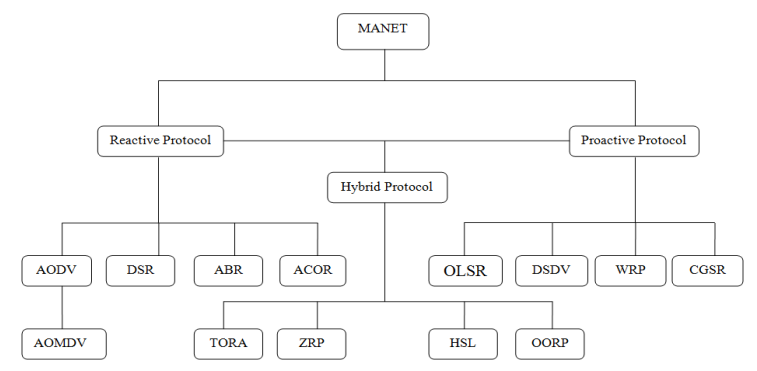

Fig 3 MANET Routing Protocol Types.

To find out awesome paths, AOMDV suppresses reproduction path requests (RREQ) at intermediate nodes. Such suppression comes in two exclusive variations, ensuing in both node (illustrated in parent (a) or hyperlink as illustrated in figure disjoint.

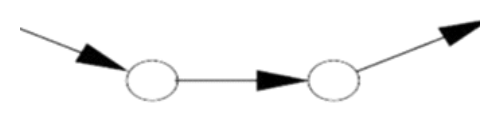

Fig 4 AODV Route Discovery

\section{Architecture of NS:}

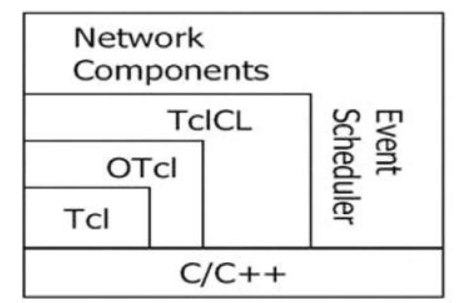

Fig 4 Architecture of NS (Basagani et al, 2004)

The NS-2 architecture (Figure 1.12) is made up of six components:

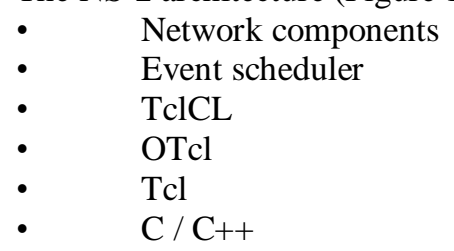

\section{IEEE802.11 Overview:}

The IEEE 802.11 standard defines two operational modes for WLANs: infrastructure-based and infrastructure less or ad hoc. When working on this mode, stations are said to shape an independent basic carrier set (IBSS) or, more absolutely, an ad- hoc network. Any station this is in the transmission range of every other, after a synchronization phase, can start communicating. No get admission to factor (AP) is required, but if one of the stations operating within the ad-hoc mode additionally has a connection to the wired community, stations forming the ad-hoc network have a wifi get entry to the net (basagni et al, 2005).

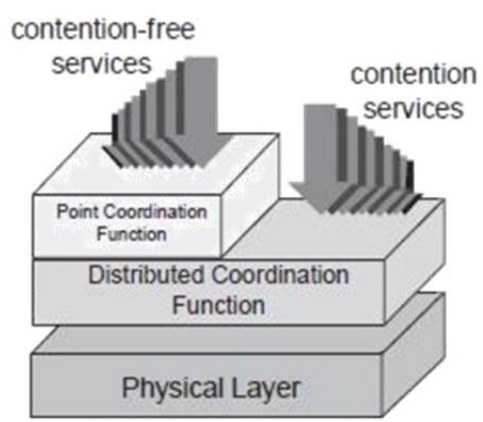

Fig 5 Layers in IEEE802.11 
Vol. 8, Issue 1, January 2019

\section{LITERATURE REVIEW}

To find out what became already been finished inside the overall performance evaluation of more than one Ad hoc Network (MANETs), a complete literature survey has been conducted and some of the findings are offered below:

Author [1] 1999 Perkins et al discussed about Ad hoc networks and challenges faced for efficient routing in this type of wireless communication as in an ad hoc network, mobile nodes communication is there with each other using multiple hop wireless links.

Author [2] 2005 Basgani et al stated that Link disjoint path option of multi-path routing protocol overall performs better than single-path or node disjoint path option of multi-path routing protocol in the presence of selfish nodes and by consider that link disjoint configuration of AOMDV, it can be a good choice to work with feedback mechanism.

Author [3] 2007 Acharjee et al described the simulation study to compare AODV, DSDV and DSDV by using a varying workload such as load, Mobility and size and it was found that at initial stages the delivery ratio for AODV and DSDV are closely same regardless of mobility changes but the delivery ratio of DSDV is very low as compare to DSDV at very high mobility levels.

Author [4] 2008 Qasim et al AODV is reactive protocol and construct route on demand and aims to reduce routing load. It uses a desk driven routing framework and destination series numbers for routing packets to destination mobile nodes and has place impartial set of rules.

Author [5] 2010 Kartik et al AODV shares DSDV's on-demand characteristics in that it also discovers routes on an as needed basis via a similar route discovery process.

Author [6] (2011) Amjada and Dojab discussed about quality of service of various on-demand protocols when simulated under small values of node variation i.e. upto 50 nodes and lesser periodic times, DSDV plays closely equivalent role as compare to other efficient protocols.

Author [7] 2012, Verma et al observed Energy efficiency as one of the main problems in a mobile ad hoc network, especially designing a routing protocol.

\section{OBJECTIVES}

To evaluate the performance comparison of different protocols, there are different simulators exists like Network Simulator, Optical networks, Matrix laboratory etc. In this work, Network Simulator (version 2.35) is used due to its easy availability and since it is also free source software. The main platform for this software is Linux and the script is written in the tool command language (TCL) with the combination of $\mathrm{C} / \mathrm{C}++$ and $\mathrm{AWK}$ files.

The present research involves Analyzing \& Evaluating the behavior AOMDV, AODV and DSDV under various scenarios like by varying number of nodes and by using different Radio Propagation Models.

Pre-simulation segment includes the process of generating the state of affairs file which explains the range of nodes, topology, and version of movement of the nodes and so on.

- $\quad$ Execution phase receives enter via the OTCL script written in preceding segment and generates a difficult form of statistics called trace file.

- $\quad$ The main intention of the submit-simulation process is to extract the useful facts from a difficult and big data file that's the previously discussed hint record generated in execution section.

As shown below in Table 4.1, simulation parameters are given. In the simulation work, one of the protocols, number of nodes and propagation channel is selected and other parameters remain constant.

Table 1. Simulation Scenario

\begin{tabular}{|l|l|}
\hline Simulation Parameters & Description \\
\hline Routing Protocols & AOMDV, AODV and DSDV \\
\hline Pause Time & 50 secs \\
\hline Propagation Models & Shadow, Two Ray and Nakagami \\
\hline Simulation time & 2060 Secs \\
\hline Traffic Type & CBR, Pareto,Expo \\
\hline Antenna Model & Omni Directional \\
\hline MAC & IEEE 802.11 \\
\hline No. of Nodes & 50, 100, 150, 200, 250 \\
\hline Channel Type & Wireless \\
\hline Que Type & Drop Tail \\
\hline Node placements & Random \\
\hline
\end{tabular}




\section{PROPOSED METHODOLOGY}

The literature survey exhibits that lot of work has been accomplished in the subject of Manets. But still there is a scope of work within the discipline of power intake, PDF and Gen throughput below various propagation models consisting of ray, shadow and Nakagami models in high mobility fashions.

- $\quad$ Firstly, we face Formulation problem, from Literature survey, it's miles clean that at decrease mobility model i.e. variety of nodes, pause time, start and prevent time, the reaction by using various protocols closely match each other but the overall performance varies as the complexity increases.

- $\quad$ The objective of the present research involves Analyzing \& Evaluating the behavior of AOMDV, AODV and DSDV under various scenarios like, Performance analysis of AOMDV, AODV and DSDV under various scenarios like by varying propagation models and number of nodes.

- $\quad$ The proposed title is "Performance Analysis of On-Demand Routing Protocols Using Different Propagation Models.

\section{RESULTS}

As shown in Figure, commands are initialized in Tool Comand Language (TCL) script in Network Simulator 2.35 to configure the AOMDV protocol for a particular set of scenarios. The performance parameters for Manets can be calculated with the aid of numerous manner and beneath various situations like by using changing any of the simulation parameter and keeping other steady that's mentioned in desk 4.1 on this work, the variables are, protocols, propagation model, number of nodes.
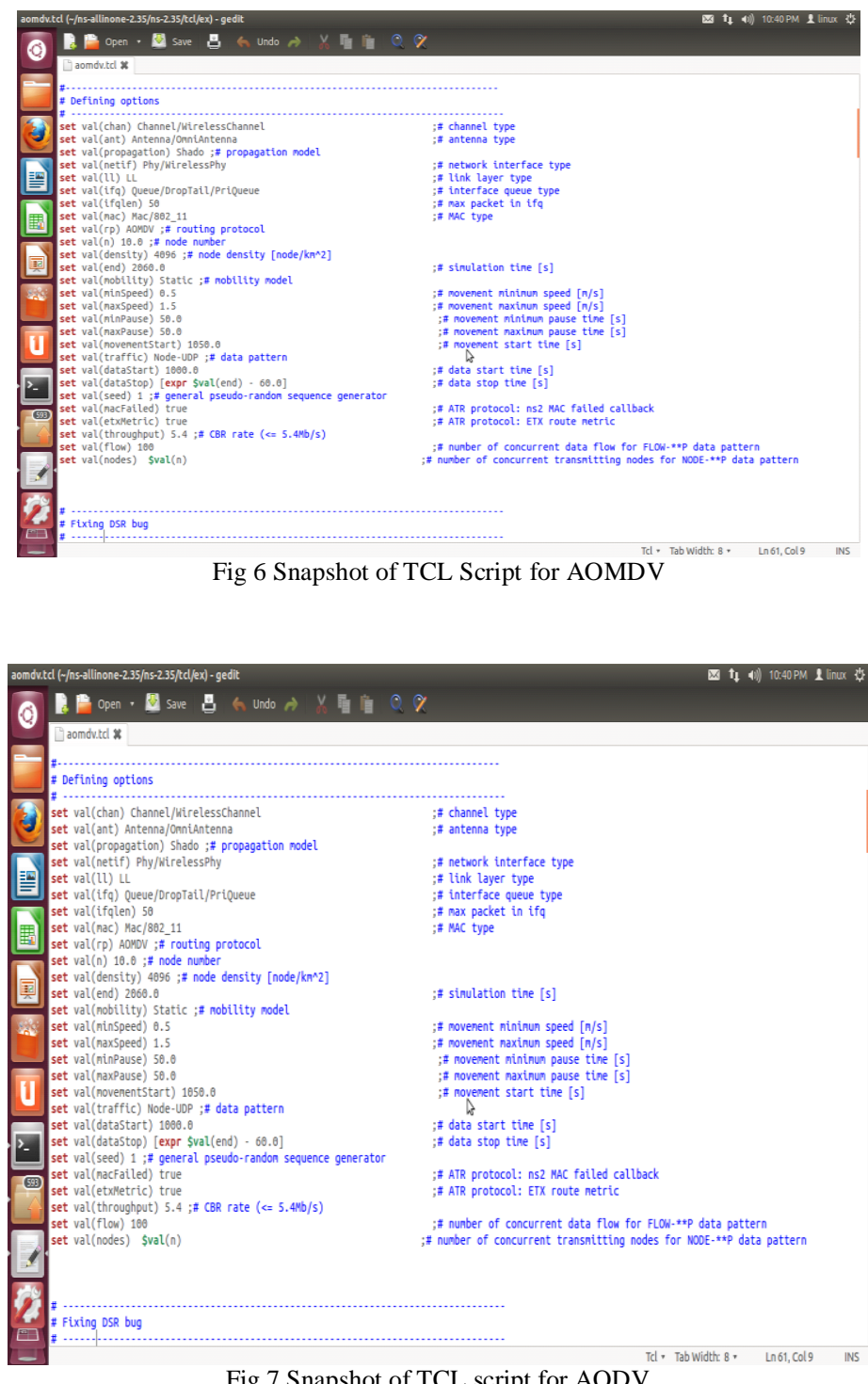
Vol. 8, Issue 1, January 2019

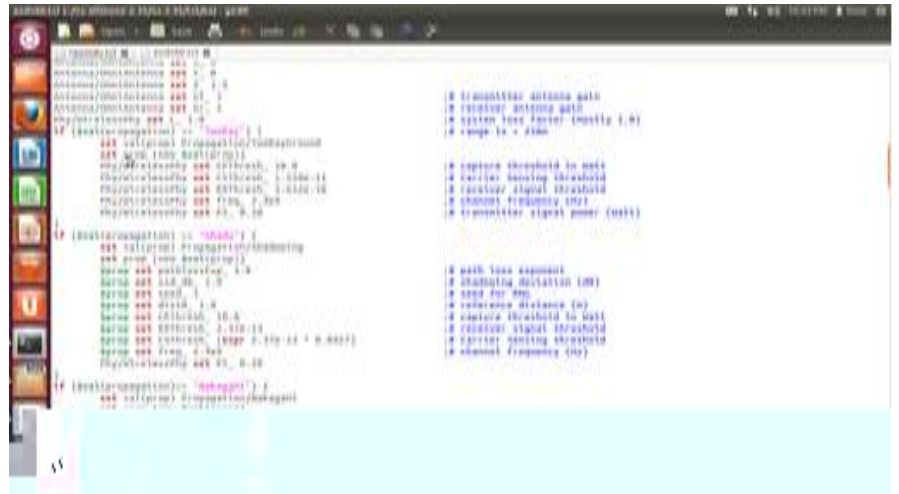

As shown in figure 4.3, TCL script is defining the kind of protocol, right here the protocol is AODV and node range is 100

Table 2 PDF v/s Nodes in Nakagami Model

\begin{tabular}{|c|c|c|c|}
\hline No.of Nodes & AODV & AOMDV & DSDV \\
\hline 50 & 1 & 1 & 0.7 \\
\hline 100 & 0.96 & 0.99 & 0.93 \\
\hline 150 & 0.96 & 0.97 & 0.91 \\
\hline 200 & 0.91 & 0.95 & 0.89 \\
\hline 250 & 0.90 & 0.95 & 0.89 \\
\hline
\end{tabular}

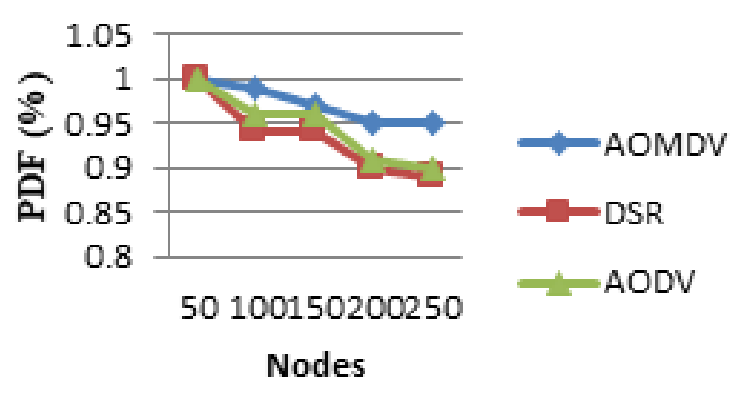

Fig8: Packet Delivery Fraction v/s No. of Nodes with Nakagami Model Case I :- PDF v/s Number of Nodes in Nakagami Model.

In Figure as shown the Packet Delivery Fraction is calculated and compared by varying the number of nodes i.e. 50 , 100,150, 200, 250 for routing protocols AODV, AOMDV and DSDV.

Table 3 Genthrouput v/s Nodes in Two Ray Model

\begin{tabular}{|l|l|l|l|}
\hline No.of Nodes & AODV (kbps) & AOMDV ( kbps) & DSDV (kbps) \\
\hline 50 & 230.01 & 235.07 & 241.94 \\
\hline 100 & 158.23 & 170.01 & 164.34 \\
\hline 150 & 134.05 & 139.87 & 132.22 \\
\hline 200 & 116.22 & 121.91 & 104.55 \\
\hline 250 & 106.25 & 112.26 & 96.76 \\
\hline
\end{tabular}

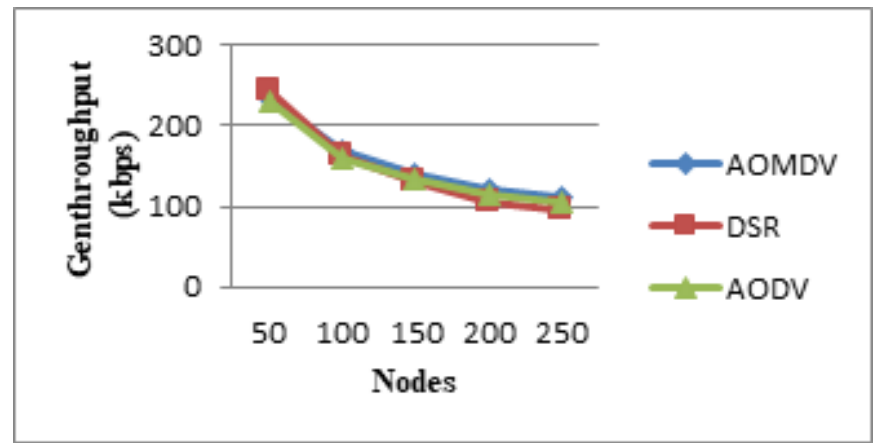


Vol. 8, Issue 1, January 2019

As shown in Figure, in Two Ray model, the DSDV has an edge over other two during initialization.

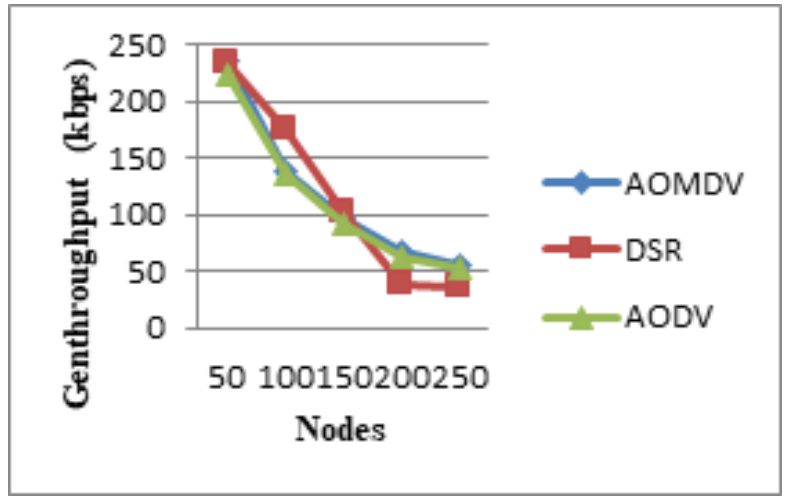

Fig 9 Genthroughput v/s No. of Nodes in Shadow Propagation Model

From above calculations, it is also observed that Nakagami and Two Ray propagations performed well than Shadow model and also since in Nakagami Model as fading parameter is also included and two Ray model is limited.

\section{CONCLUSION AND FUTURE WORK}

Simulation and evaluation of the 3 reactive protocols AOMDV, AODV and DSDV in specific simulation situations and observing their conduct in phrases of 3 enormous parameters i.e. packet shipping fraction, and gen throughput in order to discover which one need to be preferred whilst the mobile ad hoc network needs to be set up for the precise duration underneath special situations. The whole simulation scenario consisting of minimum 50 and maximum of 250 nodes is created by writing the OTCL script in NS-2 (version 2.35) and analyzing the parameters Packet delivery ratio, and Gen throughput with the help of generated graph.

In this research work, the performance comparison between three reactive protocols using CBR traffic model. More work can be possible with other different traffic models and also further research is possible in hybrid protocols comparing with active and reactive protocols and in complex mobility models where best of Active and Proactive protocols are present.

\section{REFERNCES}

[1] Acharjee, U., K., Ahmed, A. and Rafique, S. (2007), "Experimential Analysis of Ad-hoc Routing Protocols Using network simulator", International Journal of Computer Science, vol. 1, 2, pp. 1-6.

[2] Amjada, M. and Dojab, M., N. (2011), "Quality of Service Routing in Mobile Adhoc Networks", International Journal of Advanced Engineering \& Application, vol. 1, 1, pp. 165-168. [3] Basagni, S., Conti, M., Giordano, S. and Stojmenovic, I. (2004), "Mobile Ad Hoc Networking”, IEEE Press, A John Wiley \& Sons, Edition 1.

[3] Bawa, O., S., and Banerjee, S. (2013), "Congestion based Route Discovery AOMDV Protocol," International Journal of Computer Trends and Technology, vol. 4, 1, pp. 54-58.

[4] Bettstetter, C. and Hartmann, C. (2003), "Connectivity of Wireless Multi-hop Networks in a Shadow Fading Environment", IEEE Transaction on Wireless Networks, vol. 11, 1, pp. 28-32.

[5] Bhavsar, C., Vegad, M., M. and Bakhru, S., A. (2012), "Performance Comparison of Stability Enhanced AODV and AOMDV Protocols for MANETs", International Journal of Emerging Technology and Advanced Engineering. vol. 2, 7, pp. 66-73.

[6] Bindral, S., H., Maakar, S., K. and Sangal, A., L. (2010), "Performance Evaluation of Two Reactive Routing Protocols of MANET using Group Mobility Model", International Journal of Computer Sciences, vol. 7, 10, pp. 38-43.

[7] Biradar, S., R.., Majumder, K., Sarkar, S., K., and Puttamadappa (2010), "Performance Evaluation and Comparison of AODV and AOMDV", International Journal on Computer Science and Engineering. vol. 2, 2, pp. 373-377. 